\title{
ASSESSING FLOOD IMPACTS IN RURAL COASTAL COMMUNITIES USING LIDAR
}

\author{
E.S. Johnson ${ }^{\mathrm{a}}$
}

\author{
${ }^{a}$ Environmental Studies Program, Bowdoin College, 6700 College Station, Brunswick, ME 04011. \\ ejohnson@bowdoin.edu
}

\section{Commission VI, WG VI/4}

KEY WORDS: coastal, flooding, lidar, transportation

\begin{abstract}
:
Coastal communities are vulnerable to floods from storm events which are further exacerbated by storm surges. Additionally, coastal towns provide specific challenges during flood events as many coastal communities are peninsular and vulnerable to inundation of road access points. Publicly available lidar data has been used to model areas of inundation and resulting flood impacts on road networks. However, these models may overestimate areas that are inaccessible as they rely on publicly available Digital Terrain Models. Through incorporation of Digital Surface Models to estimate bridge height, a more accurate model of flood impacts on rural coastal residents can be estimated.
\end{abstract}

\section{INTRODUCTION}

\subsection{General Instructions}

Coastal communities will face an array of challenges with an increasing frequency of storm events and rising sea levels. In the United States it is predicted that 3.7 million coastal residents will be impacted by a 1 meter rise in sea level (Strauss 2012). In addition, 100 year storm events are now predicted to occur in 10 year cycles (Tebaldi 2012, Johnston 2014).

Although urbanized coastal areas face distinct impacts from climate change, rural coastal communities are particularly vulnerable to factors associated with the impacts of storms on infrastructure. Infrastructure such as transportation networks plays a key role in supporting coastal communities. Damage to transportation systems can have a myriad of impacts including hampering emergency response efforts particularly during storm events (Johnston 2014). Many rural residents have fewer options for evacuation with longer travel times to hospitals and shelters. Coupled with fewer resources to address infrastructural impacts, planning for storm events in rural coastal areas may require special consideration (Liu 2015, Murphy 2014). Impacts are particularly problematic for coastal areas where older residents, those above 65 years of age, represent a larger proportion of the overall population. Older populations may have reduced ability to respond to emergency conditions, limited financial resources to prepare for natural disasters such as flood events, and tend to be reluctant to evacuate (Krawchenko 2016).

Maine, a coastal state in the continental US, is projected to see level rise ranging from 1.14 to 1.56 meters within 50 years. With one of the highest levels of tidal change in the continental US, and with 100 year events predicted to occur between every 5 and 20 years, Maine faces a distinct set of challenges (Tebaldi 2012). Many rural fire departments in coastal communities are staffed by volunteers and locations of emergency service providers such as police and ambulance are not located within each community, but at county and regional levels. As a result response times may be further hampered by flood events that render roads impassable and sections inaccessible. Along Maine's coast in the continental US, an additional challenge is an aging population with the inherent challenges of responding to emergency situations for this population.

Resilience and adaptation planning have highlighted the need for better models to effectively plan for emergency response (Wilby 2012, Tang 2013). Development of models that identify key infrastructure at risk during flood events enables communities to plan more effectively for flood events and develop mitigation strategies (Oh 2010). One source of data is FEMA's FIRM Maps that provide updated information on flood risks to communities. These maps are widely available in North American and identify areas subject to flooding during 100 year flood events. Base Flood Elevation (BFE) is the elevation for 100 year flood events in inland areas (AE zones) and includes elevation of wave crests above still water elevations in coastal areas (VE Zones) (Tang 2013, Tebaldi 2012). The use of FEMA flood maps has been increasingly incorporated into emergency planning for flood events and assessment of damage after 100 year storm events (Patrick 2015, Oh 2010, Xian 2015).

However, many models fail to incorporate bridge elevations into transportation models, relying on DTMs that do not always include bridge locations (Johnston 2014, Tang 2013). One challenge is the lack of readily available data on bridge locations and heights. Having a means of more easily including bridge elevations is essential for better emergency planning (Tang 2013).

Increasingly, lidar is being used to refine models of flooding impacts. Lidar has been used to develop higher precision Digital Elevation Models (DEMs) through the use of Digital Terrain Models (DTMs) for modelling flood levels. Lidar data has also been used to develop Digital Surface Models (DSMs) to analyse impacts of bridge locations and heights on water flow during flood events, and can be used to more effectively align simulated impacts with measured impacts of flooding (Meesuk 2015, Abdullah 2012). Although the incorporation of DSMs has been shown to provide a better simulation model for urban flooding, few studies have been shown to incorporate DSMs for identifying bridge locations and heights to enhance transportation models in rural areas (Meesuk 2015, Abdullah 2012, Camill 2012, Johnston 2014). 
This study examines the implications of modifying transportation analyses through the inclusion of DSMs to identify bridge locations and elevations in order to improve overall transportation network models associated with storm events.

\section{METHODS}

\subsection{Study Area}

Maine has the second longest tidal shoreline of any continental state in the United States. Coastal Maine towns, those communities located directly adjacent to the coast and therefore most impacted by rising sea levels and coastal storms, comprise $35 \%$ percent of the state's overall population. Although coastal towns represent only $7 \%$ of the total land mass of Maine, they contain over $20 \%$ of the state's road network due in part to the peninsular nature of coastal communities. The study area includes an analysis of 6 coastal counties. York County, representing 8 coastal communities, was excluded from the study, as FEMA FIRM maps were not available at a county level. Due to the processing requirements of generating DSMs, a subset study area, the Midcoast region, representing 12 communities, was selected to examine the implications of integrating DSMs into a transportation network analysis of flood impacts.

\subsection{Network analysis of emergency response}

For the purposes of modelling emergency response, locations of emergency responders including police and fire departments and ambulance services were assembled at a state wide level. A road network was created from the Maine Office of GIS emergency road data. Using ArcGIS 10.3 Network Analyst, areas within a 60 minute response time of emergency responders were generated for all towns within the study area as a baseline data set for comparison. The location of FIRM 100 year AE, A, VE flood zones were used to extract road sections subject to inundation during a 100 year storm event. To examine the implications of integrating bridge locations in modelling emergency response during flood events, three scenarios were analysed. Scenario 1 examined a standard approach to modelling flood conditions that bases network models on Digital Terrain Models and assumes all road portions falling within a flood zone would be inundated during a 100 year storm event. Scenario 2 modifies emergency response times by incorporating bridge locations. Scenario 3 incorporates DSMs that provide bridge elevations to further refine the network analysis. Scenario 3 is provided for the Midcoast area. To examine impact on coastal populations, locations of buildings were incorporated. For each scenario the number of buildings that are inaccessible during a flood event were generated. Additionally, response times from the closest emergency response center were created for all three scenarios.

Scenario 1 is based upon elevations derived from DTMs. For this scenarios, a network analysis was conducted that incorporated inundated roads as barriers to identify coastal areas that would be inaccessible during a 100 year flood event. Scenario 2 incorporated bridge locations as a vector data set. Bridge locations are provided at a state level in Maine, but only as point locations and do not include information on bridge dimensions and elevations. There are 1,240 bridges within the study area. For Scenario 2, an average bridge length of 100 meters was selected. Flood zones were modified to remove bridge locations using a 100 meter buffer. The network analysis was run with modified flood zones to identify roads that would still be inaccessible with inclusion of bridge locations.

\subsection{Integration of Digital Surface Model (DSM)}

To examine the validity of developing a standardized model for integration of bridge locations to assess implications for emergency response, a subset of the study area was selected to examine relationships between this standardized analysis (Scenario 2) and the integration of DSM (Scenario 3). Currently, digital elevation models (DEM) are available as DTMs at a state wide level. Using ArcGIS 10.3 3-d Analyst, a DSM was created from las data sets for the Midcoast study area. There are 128 bridges within this study area. Using aerial imagery, footprints of bridge locations were digitized. Zonal statistics for minimum and maximum elevations for both DTMs and DSMs were generated for each bridge footprints. Elevations of bridges were compared with the BFE of $\mathrm{AE}$ and $\mathrm{VE}$ zones to identify bridges that may be inundated in a 100 year flood event. The network model was modified as a result and the location of inaccessible areas, building locations, and response times were generated for Scenario 3 .

\section{RESULTS AND DISCUSSION}

The results of the three scenarios are presented in Table 1 for both the study area and the Midcoast area. Results for the study area are presented for scenarios 1 and 2, while results for the subset Midcoast study are presented for Scenarios 1-3.

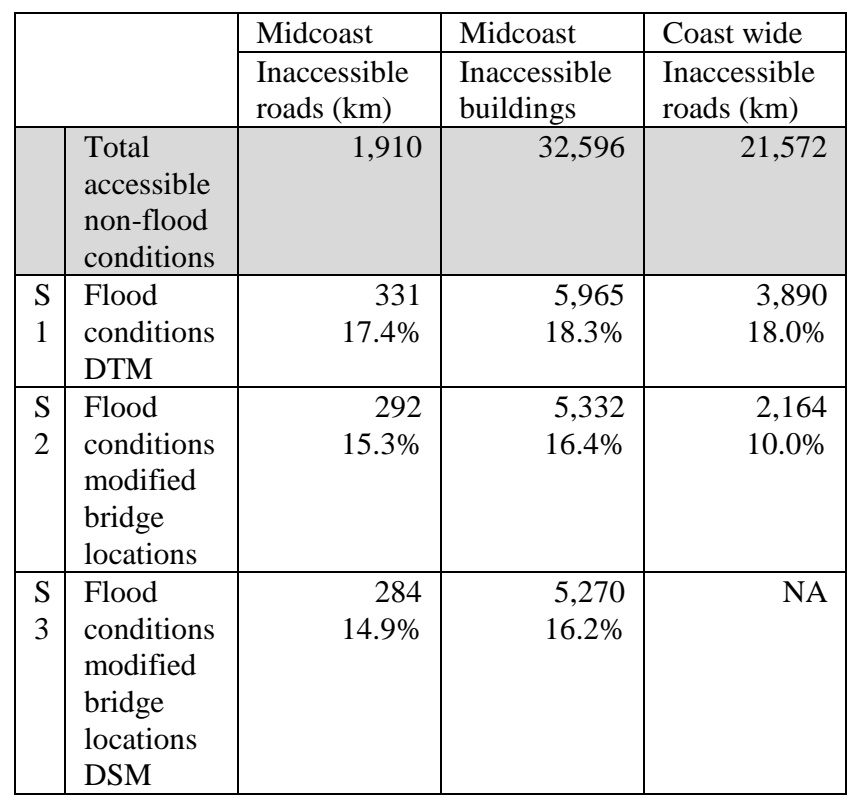

Table 1 - Inaccessible roads ( $\mathrm{km}$ ) and number of inaccessible buildings in flood conditions each scenario

During 100 year storm events, a significant portion of coastal roads may become inaccessible to emergency responders. At a statewide level there is an important difference between DTM based network models and models that are modified to include bridge locations. As shown in Table 1, models of transportation impacts using traditional DTM may overestimate the number of regional roads and associated structures that may be 
inaccessible to emergency responders during flood events. Within the study area, a model based up on DTMs (Scenario 1) estimated $17 \%$ of roads network may be inaccessible during a storm event. Modifications of network analyses using bridge elevations (Scenario 2) could reduce the number of inaccessible roads during a flood event to $10 \%$. As bridge locations do not necessarily imply sufficient height to overcome flood conditions, the use of lidar for the generation of DSMs can provide critical information on bridge height elevations.

The location of inundated roads emphasizes the challenges faced by rural peninsular coastal communities. Figure 1 shows the location of roads that are identified as inaccessible for a model based upon DTMs (Image A). Under Scenario 1, roads (marked in green) that are identified as inaccessible using DTMs may not be subject to flooding, once bridge locations and elevations are incorporated into network models. Image B shows the results of a model based upon DSM and shows roads (in black) that may still be inaccessible during 100 storm events due to road inundation, even with the incorporation of DSMs for identifying bridge locations and elevations. In Image B, roads in green are areas that would not likely be inaccessible based upon a DSM derived model.

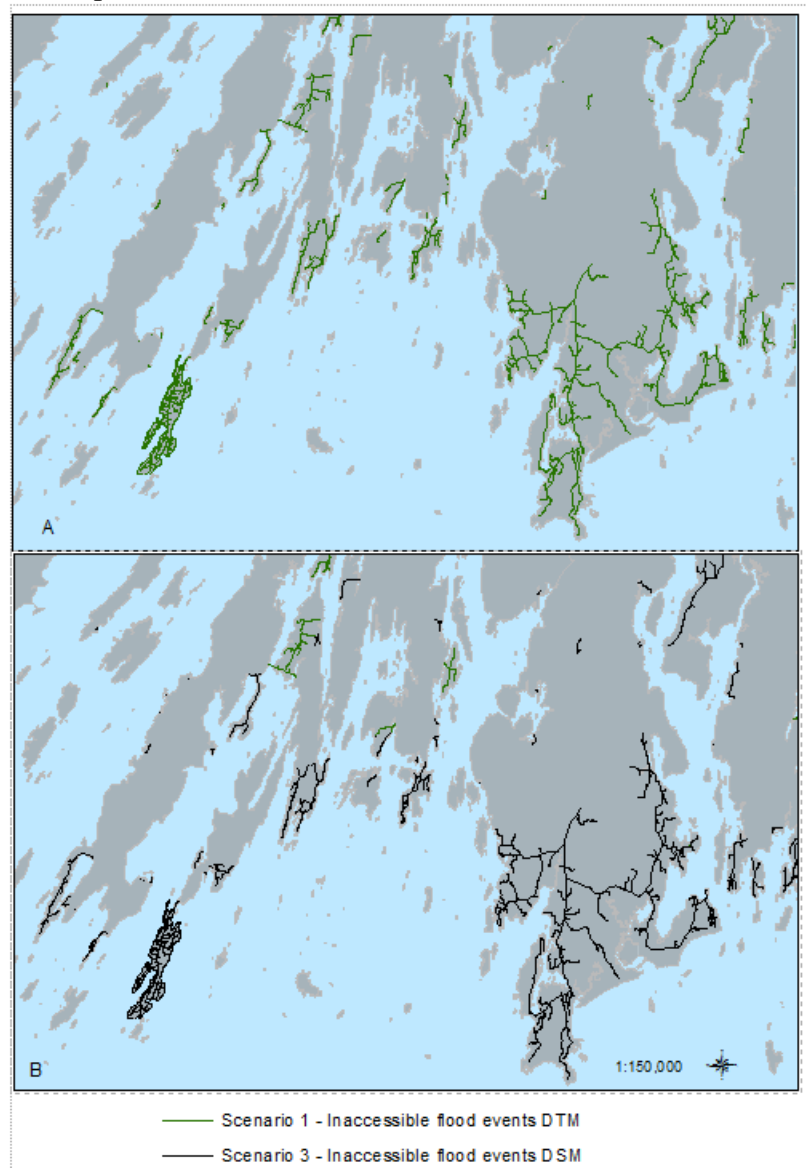

Figure 1 - Inundated roads under Scenarios 1 and 3

A second important dimension of planning for flood events, is identification of structures where coastal residents live who may find themselves unable to evacuate during flood conditions. Figure 2 presents the location of coastal residences (purple) that would be identified as being inaccessible during a 100 year storm event, using DTMs. When the analysis is modified to use DSMs to incorporate bridge locations and elevations, while residences marked in purple were still inaccessible, residences marked in black no longer identified as inaccessible during a 100 year storm event. In developing emergency response plans, increased knowledge on actual areas within a community that are more likely to experience infrastructural impacts will enable more efficient emergency response planning.

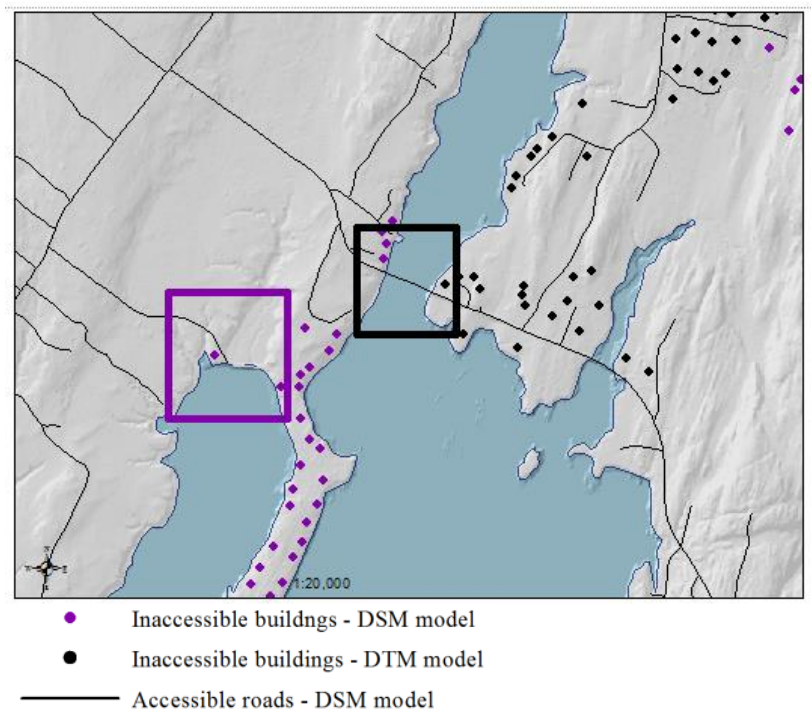

Figure 2 - Locations of inaccessible buildings

Figure 3 illustrates the differences between DTMs and DSMs in modelling road networks. In images $\mathrm{A}$ and $\mathrm{B}$, a coastal road shown in the purple box in Figure 2, is apparent in both the DSM (Image A) and DTM (Image B). In images C and D, a bridge (shown within the black box in Figure 2) is not visible in the DTM but is evident in a DSM (Image D).

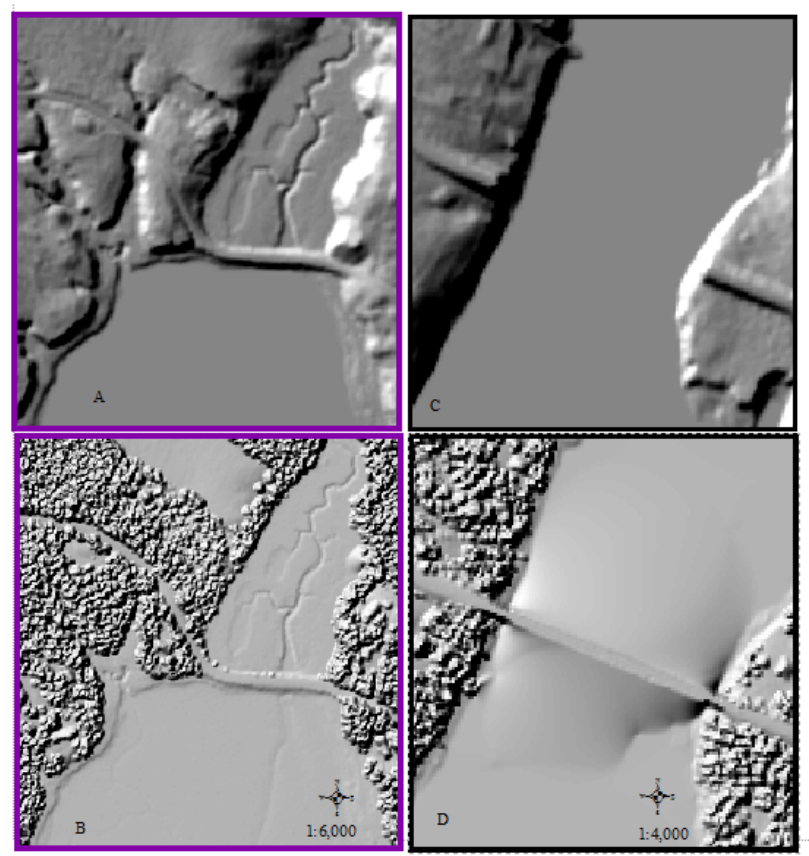

Figure 3 - Inundated road sections with Digital Terrain Model and Digital Surface Model.

The use of spatial analysis coupled with DSMs can provide an assessment of the likelihood that a bridge may still be inundated during a storm event. Table 2 provides the results of zonal statistics for road and bridge sections shown in Figure 3. BFE for the AE zone (shown in Figure 2, Images A and B) and BFE 
for the VE zone (shown in Figure 2, Images $C$ and D), show that the road in Image A and B will likely be inundated during a 100 year storm event as the BFE is greater than the elevation of the road in either the DSM or DTM model. In images C and D, the BFE is less than the elevation of the bridge based upon the DSM and therefore not subject to inundation.

\begin{tabular}{|l|c|c|}
\hline & Images A and B & Images C and D \\
\hline $\begin{array}{l}\text { FIRM AE/VE } \\
\text { BFE (m) }\end{array}$ & 3.6576 & 4.8768 \\
\hline DSM Min (m) & 2.55 & 11.85 \\
\hline DTM Min (m) & 2.96 & 0 \\
\hline
\end{tabular}

Table 2 - Minimum elevation for DSM and DTM for inundated road sections

A second component of integration of DSMs into emergency planning is the ability to provide more accurate data for communities on key areas needing infrastructural improvements. Figure 2, Images A and B, show that inundation of this portion of road may have an important impact on accessibility of coastal residences during a 100 year flood event. As a result communities can target these areas for infrastructural improvements as part of resilience planning.

A second component of emergency planning is response time. Predicting accurate response times can also be impacted by accuracy of DEMs used in network modelling. Image A in Figure 4 shows response times for network models that incorporate DTMs. In this case, response times fall in the range of 11-18 minutes. When the network model is modified to incorporate DSMs (Image B) to include bridge locations and elevations, the revised transportation analysis show response time falling closer to 8-10 minutes.

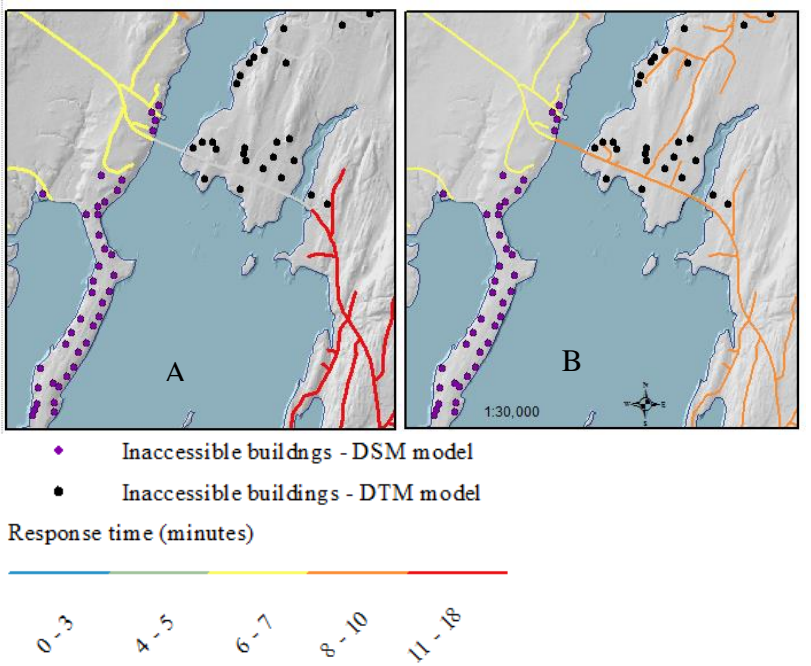

Figure 4 - Response Times

\section{CONCLUSION}

As 100 year storm events become more frequent, transportation systems will be impacted for even routine transportation needs. Accurate models of flood impacts are critical for rural regions facing a lack of capacity for addressing infrastructural needs. Generation of more accurate models based upon DSMs can inform local emergency agencies on areas requiring particular focus during a storm event. Modelling location of inundated roads also provides important insights on the implications for evacuation routes for coastal residents during storm events. Further, accurate models can provide opportunities to target needed infrastructural improvements such as raising roads and replacement of culverts.

This study helps to inform the need to identify areas within a community that are most likely to be impacted during flood events, enabling the effective deployment of emergency responders. Accurate models are particularly important in rural areas that may face resource capacity challenges due to dispersed location of emergence response centers and limited available emergency responders. Having better information on areas that may or may not be impacted by flooding enables better planning in the likelihood that an emergency responder must respond to an emergency during a flood event.

Although the use of lidar in generating DSMs to refine network analysis shows promise, there are challenges in applying this approach to a large scale, such as coastal Maine. Data gaps and inaccuracies such as bridge locations provide challenges in developing a simple methodology for extracting bridge locations and elevations based upon DSMs. Generation of DSMs can be computationally intensive. Beyond these challenges, the incorporation of DSMs in evaluating infrastructural impacts of flooding events can provide better models for emergency management and adaptation strategies for responding to increased coastal flooding in response to climate change.

\section{ACKNOWLEDGEMENTS}

The author would like to thank Tyler DeAngelis, Jeremy Dobkin, Colton Hall and Ezra Duplissie-Cyr for data contributions to this study.

\section{REFERENCES}

Abdullah, A., Z. Vojinovic, R. K. Price, and N. a a Aziz. 2012. "A methodology for processing raw LiDAR data to support urban flood modelling framework." Journal of Hydroinformatics 14 (1),75-75.

Camill, Philip, Maryellen Hearn, Krista Bahm, and Eileen Johnson. 2012. "Using a boundary organization approach to develop a sea level rise and storm surge impact analysis framework for coastal communities in Maine." Journal of Environmental Studies and Sciences 2 (2),111-130.

Johnston, A., P. Slovinsky, and K. L. Yates. 2014. "Assessing the vulnerability of coastal infrastructure to sea level rise using multi-criteria analysis in Scarborough, Maine (USA)." Ocean and Coastal Management 95,176-188.

Krawchenko, Tamara, Janice Keefe, Patricia Manuel, and Eric Rapaport. 2016. "Coastal climate change, vulnerability and age friendly communities: Linking planning for climate change to the age friendly communities agenda." Journal of Rural Studies 44,55-62. 
Liu, Hua, Joshua G. Behr, and Rafael Diaz. 2015. "Population vulnerability to storm surge flooding in coastal Virginia, U.S.A." Integrated Environmental Assessment and Management 9999 (9999), 1-10

Meesuk, Vorawit, Zoran Vojinovic, Arthur E. Mynett, and Ahmad F. Abdullah. 2015. "Urban flood modelling combining top-view LiDAR data with ground-view SfM observations." Advances in Water Resources 75,105-117.

Murphy, Brenda L., Gregory S. Anderson, Ron Bowles, and Robin S. Cox. 2014. "Planning for disaster resilience in rural, remote, and coastal communities: moving from thought to action." Journal of emergency management (Weston, Mass.) 12 (2):105-20.

Oh, Eun Ho, Abhijeet Deshmukh, and Makarand Hastak. 2010. "Disaster impact analysis based on inter-relationship of critical infrastructure and associated industries: A winter flood disaster event." International Journal of Disaster Resilience in the Built Environment 1 (1),25-49.

Patrick, Lesley, William Solecki, Klaus H. Jacob, Howard Kunreuther, and Guy Nordenson. 2015. "New York City Panel on Climate Change 2015 Report Chapter 3: Static Coastal Flood Mapping." Annals of the New York Academy of Sciences $1336(1), 45-55$.

Strauss, Benjamin H., Remik Ziemlinski, Jeremy L. Weiss, and Jonathan T. Overpeck. 2012. "Tidally adjusted estimates of topographic vulnerability to sea level rise and flooding for the contiguous United States." Environmental Research Letters 7 (1), 014033-014033.

Tang, Han Song, Steven I. Jy Chien, Marouane Temimi, Cheryl Ann Blain, Qu Ke, Liuhui Zhao, and Simon Kraatz. 2013. "Vulnerability of population and transportation infrastructure at the east bank of Delaware Bay due to coastal flooding in sealevel rise conditions." Natural Hazards 69 (1), 141-163.

Tebaldi, Claudia, Benjamin H. Strauss, and Chris E. Zervas. 2012. "Modelling sea level rise impacts on storm surges along US coasts." Environmental Research Letters 7 (1), 014032014032 .

Wilby, Robert L., and R. Keenan. 2012. "Adapting to flood risk under climate change." Progress in Physical Geography 36 (3), 348-378.

Xian, Siyuan, Ning Lin, and Adam Hatzikyriakou. 2015. "Storm surge damage to residential areas: a quantitative analysis for Hurricane Sandy in comparison with FEMA flood map." Natural Hazards 79 (3), 1867-1888. 